\title{
Is There a Relation Between Caregiver Burden and Cognitive Dysfunction in Huntington's Disease?
}

\author{
M. M. Schumacher-Kuiper (D) A. M. van Loon - C. F. W. Peeters • \\ M. R. Ekkel - C. M. P. M. Hertogh • R. B. Veenhuizen
}

Received: 10 July 2020/Accepted: 3 October 2020/Published online: 14 October 2020

(C) The Author(s) 2020

\begin{abstract}
Caring for a family member with Huntington's disease (HD) can be seriously burdensome. Cognitive and neuropsychiatric symptoms that are part of HD can impact the quality of life of caregivers. Therefore, we investigated the relationship between caregiver burden, cognitive impairment and patient characteristics. A retrospective cross-sectional study was performed on 33 adult HD-outpatient-caregiver dyads. We assessed caregiver burden and cognitive functioning of the included patient on the same day
\end{abstract}

Electronic supplementary material The online version of this article (https://doi.org/10.1007/s40737-020-00202-8) contains supplementary material, which is available to authorized users.

M. M. Schumacher-Kuiper ( $\square$ )

Gerion, Amsterdam UMC, Vrije Universiteit Amsterdam, De Boelelaan 1109, Amsterdam, The Netherlands

e-mail: schumachermaaike@gmail.com

M. M. Schumacher-Kuiper · R. B. Veenhuizen

Noorderbreedte Care Group, Leeuwarden, The

Netherlands

A. M. van Loon - M. R. Ekkel · C. M. P. M. Hertogh ·

R. B. Veenhuizen

General Practice and Old Age Medicine, Amsterdam

UMC, Vrije Universiteit Amsterdam, De Boelelaan 1117,

Amsterdam, The Netherlands

C. F. W. Peeters

Epidemiology and Biostatistics, Amsterdam UMC, Vrije Universiteit Amsterdam, De Boelelaan 1117, Amsterdam, The Netherlands with the MCSI and MoCA respectively. For statistical analysis, we performed a network analysis and used descriptive statistics to describe our study sample. Caregivers scored on average 13.5 out of 26 points on the MCSI. The scores on the MoCA of the HD patients varied from 9 to 30 and was on average 22. Our network analysis demonstrated an indirect relationship between cognitive functioning and caregiver burden, in which CAG repeat length and the time since HD has been diagnosed are the primary mediators. We found a negative association between CAG repeat length and cognitive functioning. Furthermore, a relationship was found between higher caregiver burden and psychotropic drug use. We observed an indirect relationship between cognitive functioning and caregiver burden using network analysis. This analysis produces comprehensible results with the variables of interest. This study sheds new light on the components that make up caregiver burden in HD.

Keywords Caregiver burden - CAG repeat . Cognition · Psychotropic drugs · Network analysis

\section{Introduction}

Huntington's disease (HD) is an autosomal-dominant neurodegenerative disease characterized by motor, cognitive and neuropsychiatric symptoms. The prevalence of HD in North Western Europe, North America 
and Australia ranges from 5.96 to 13.7 per 100,000 persons [1]. HD is caused by an elongated CAG trinucleotide repeat on the short side of chromosome 4 encoding for the HTT (Huntingtin) protein [2].

Caring for someone with HD can be complex and challenging. Especially, given the progressive trajectory of $\mathrm{HD}$, the person afflicted requires more intensive care over time. It is suggested that the impact of HD on the quality of life of caregivers is more significant than in other neurodegenerative diseases [3]. The theme which was most widely discussed by caregivers in the literature according to the review on the impact of HD on caregivers by Domaradzki were their negative experiences with health and social care services, such as insufficient HD care and housing options [4]. The hereditary character of HD burdens caregivers with the fear that the disease develops in other family members, notably the children. Another burdensome aspect which Domaradzki highlights, is the difficulty maintaining a meaningful relationship with the patient because of HD symptoms and the decreasing reciprocity. The struggle with the changing roles over the course of the disease and the struggle with the increasing need for care also emerged in interviews with caregivers [5]. In semidirected focus groups, caregivers addressed emotional well-being, dealing with personal burden (such as losing their own identity and their responsibility of caring), the struggle with the practicalities of caregiving and the social support as having a negative impact on their quality of life [6]. Additional burdening factors for caregivers are the fact that the majority of skilled care is provided by these caregivers and the lack of knowledge on the trajectory of HD, according to the review by Tarolli [3].

Furthermore, HD-associated cognitive dysfunctions have a great impact on the quality of life of caregivers as well [7]. About 54\% of HD caregivers pointed out the combination of behavioral and cognitive symptoms as most impactful [8]. Detrimental to the quality of life of caregivers are symptoms such as executive dysfunction and the cognitive decline in general. Moreover, the cognitive changes can make it harder for caregivers to maintain a normal household [9]. For example, interviewed caregivers indicated that it can be hard for them to combine the caregiving role and their role as employees [10].

In general the cognitive performance of $\mathrm{HD}$ patients worsens as the disease progresses [11]. Early in the disease cognitive symptoms such as impairment of attention, memory, psychomotor speed, emotional recognition and visuospatial processing can occur. Executive dysfunction, deficits in problem solving and cognitive inflexibility are also exhibited by patients with early $\mathrm{HD}$, whereas domains such as language comprehension, spatial awareness and orientation, and semantic memory remain relatively intact in the early stages [12, 13]. Vinther-Jensen et al. found in an outpatient setting that $14 \%$ of premanifest geneexpansion carriers and $77 \%$ of the motor manifest HD patients show cognitive impairments [14]. Thus, cognitive decline can be seen in HD patients who do not show motor symptoms yet [12-15].

Until now, drugs have been found to be ineffective to treat cognitive impairment in HD. Dickey and La Spada compiled an overview of the pharmacological treatment of cognitive impairment in HD and found none of the different classes of drugs significantly improved cognition [15]. Moreover, any psychoactive drug might impair cognitive functioning [16]. In 2011, a study in the United Kingdom was performed to identify the central nervous system (CNS) drugs that are prescribed to patients with HD in primary care [17]. Of the psychotropic drugs, antidepressants (49\%), antipsychotics (38\%) and hypnotics/anxiolytics $(36 \%)$ were prescribed most often. The use of these drugs was higher amongst HD patients in nursing facilities, i.e. antipsychotics $62 \%$ and anxiolytics $59 \%$ [18]. However, to the best of our knowledge, no study has looked into the effect of psychotropic drugs use on caregiver burden in HD. We hypothesized a relation between cognitive dysfunction, psychotropic drug use and caregiver burden.

Thus, in this retrospective study we explored the relationship between caregiver burden and cognitive functioning of HD patients in an outpatient setting. The novelty in this study is the patient's impaired cognition and caregiver burden were tested directly in patient-caregiver dyad at the same day, which enables to collate the level of caregiver burden with the cognitive impairment at that moment. According to our hypothesis, cognitive dysfunction is related to a higher burden experienced by the caregiver. Secondly, the use of psychotropic drugs and other patient characteristics were investigated as possible influencers of this relationship. Here, we applied networkanalysis to investigate the relationships between our variables of interest. Network analysis has emerged as 
a key technique in sociology and has also been applied successfully in biology, information science and social psychology. Recently, it has also been used to study the relations of psychopathology, because the interactions between symptoms can be understood as a network, in which symptoms are nodes and causal interactions between symptoms are connections between nodes [19]. Even though the symptoms are phenomenologically distinct, they are causally homogeneous because they are causal effects of the same disease. From this perspective, brain disorders such as HD might also be understood as networks of interacting symptoms.

\section{Methods}

\section{Setting}

In the northern part of the Netherlands, there is an outpatient HD clinic, situated in a long-term care facility, which delivers multidisciplinary treatment and care to patients with HD and their caregivers. On average, the HD patient and his/her caregiver visit this clinic biannually. On the day of the visit the patient and a close relative or caregiver are separately assessed by an elderly care physician, a psychologist, an occupational therapist, a speech and language therapist, a social worker, a physiotherapist and a case manager. This setup has been proven feasible and appreciated by patients, caregivers and health care professionals and is in concordance with the international standards of care [20,21]. When patients and caregivers visit the facility for the first time, an information brochure is given which states their pseudonymized data can be used for research purposes. They can refuse this at any time, the refusal will be noted in the electronic file and patients will be excluded from participation in research.

\section{Participants}

Patients were recruited from the above-mentioned outpatient HD clinic $(N=80)$. Patients were excluded from this study when they met the following criteria: under 18 years of age $(N=1)$, assisted living $(N=7)$, living without the primary caregiver $(N=13)$ and no visit to the HD clinic in $2018(N=10)$. Four patients did not undergo testing during the visit at the HD clinic due to severe physical or cognitive limitations. Because of the main research question, we excluded patient-caregiver dyads when the cognitive performance via the Montreal Cognitive Assessment (MoCA) and the caregiver burden via the Modified Caregiver Strain Index (MCSI) were not assessed at the same visit $(N=12)$. When either or both the MoCA and MCSI were assessed during multiple visits, the last visit was included. In total we included 33 patients and their caregivers in this study.

\section{Measures}

The measures used in this study were part of the standard treatment and care protocol for patientcaregiver dyads.

To assess caregiver burden, the MCSI was applied. The MCSI allows a more quantitative view of the burden of caregiving and consists of thirteen questions with three response categories (yes, sometimes or no). According to Thornton and Travis, the MCSI is a better instrument for measuring the burden of caregiving than the CSI (Caregiver Strain Index) [22]. Following Thornton and Travis, we converted the responses into numerical categories $(2=$ yes, $1=$ sometimes, $0=$ no) to enable an ordinal numerical measurement of the caregiver burden, resulting in a maximum score of 26 points. A higher score indicates more caregiver burden.

The MoCA was used to assess the cognitive functioning of the patients. The MoCA was developed in 1996 to screen patients with mild cognitive dysfunction and translated to the Dutch language in 2004 [23]. The maximum score is 30 points, indicating high cognitive functioning. A total score over 26 is considered normal. The tested domains include delayed recall, executive function, attention, naming, abstraction, orientation and language.

\section{Assessment}

The records of the included patients were screened for the following information:

- Basic patient characteristics: age, gender, years of education (12 years or less and more than 12 years).

- Disease-related characteristics: number of years since HD diagnosis, CAG repeat length, 
comorbidities, medication (divided into psychotropic drugs and other drugs. 'When required' medication was excluded).

- Patient measures: TFC (Total Functional Capacity, part of the Unified Huntington Disease Rating Scale, UHDRS) [24], MoCA.

- Caregiver characteristics: gender, relation to the patient.

- Caregiver measures: MCSI.

\section{Statistical Analysis}

Descriptive statistics were calculated for the characteristics and outcome measures. Because of the large variation in comorbidities, this variable was excluded from further statistical analysis.

To map and analyze the relations between MoCA, MSCI and other variables, we employed the network analysis. Network analyses can be utilized to clarify relations between a variety of variables. The analysis provides insight how the variables are linked to each other in a highly graphic way. By representing relationships between variables (e.g., correlations) as edges between nodes, important structures can be detected that are hard to extract by other means. In general, network analysis enables the researcher to represent complex statistical patterns in clear pictures, without the need for data reduction methods [25]. Furthermore, by assessing the centrality scores the most important variables in the network can be identified.

The network extraction is based on graphical modeling, for which the graph expresses the conditional (in)dependencies between the features of interest. Specifically, ridge estimation of the inverse correlation matrix (i.e., scaled partial correlations) was performed followed by support determinations based on a local false discovery rate (LFDR) procedure [26]. This approach enables network modeling when the number of features is high relative to the number of observations and when strong collinearity is present between the features. Moreover, it allows for probabilistic control over the support determination and allows for the incorporation of prior information. The resulting network can be interpreted as a conditional independence graph in which the nodes represent the variables and the edges connecting the nodes represent substantive partial correlations. Hence, linkage between two features in the network implies that conditioning on the remaining features cannot explain the association between these two features. The ridge estimation approach is preceded by a nonparanormal transformation and subsequent standardization of the data [27]. In this approach a penaltyparameter determines the rate of shrinkage towards a target matrix [26]. The target matrix was taken to be the uninformative identity matrix and the optimal penalty parameter was determined by 5 -fold crossvalidation of the negative log-likelihood of the model. An edge was considered to be present (or, analogously, a partial correlation was considered to be nonnull) when the posterior probability of being present (based on the LFDR) equalled or exceeded 0.8. For the visualization of the network we choose the KamadaKawai algorithm [28].

\section{Results}

The demographic and clinical characteristics of the patients and caregivers are shown in Table 1. Our study sample consisted of a more or less equal number of men and women and patients were on average 53 years old. On average, the TFC was 7 which indicates that the patients are in the third stage of HD. The third stage entails they are no longer employable and need support with their finances, domestic chores and planning of their daily life.

On average, caregivers experienced their burden as moderate (13.5 points out of 26 points). Yet, we observed a lot of variations in the answers of the individual questions. For example, most caregivers (70\%) found it upsetting to find their loved one had changed so much, whereas only $6 \%$ was worried about their financial situation. The answers to the thirteen MCSI questions of the 33 caregivers are given as supplementary data.

Overall, cognitive functioning as assessed with the MoCA was marginally disturbed (mean: 22) in our sample, since above 26 is considered normal. Yet, we observed quite some variation within our sample, the highest total score was 30 points and the lowest was only 9 points. Furthermore, the domains were differently affected. Patients mostly scored the highest on the domains naming and orientation that were still relatively intact. However, language, delayed recall and executive functioning were on average strongly 
Table 1 Demographic and clinical characteristics of HD patients and their caregivers

\begin{tabular}{|c|c|c|}
\hline & Patients $(N=33)$ & Caregivers $(N=33)$ \\
\hline \multicolumn{3}{|l|}{ Gender, $N(\%)$} \\
\hline Male & $17(51.5)$ & $16(48.5)$ \\
\hline Female & $16(48.5)$ & $17(51.5)$ \\
\hline Age in years, mean (SD) & $53.4(11.9)$ & \\
\hline \multicolumn{3}{|l|}{ Education, $N(\%)$} \\
\hline$\leq 12$ years & $15(45.5)$ & \\
\hline$>12$ years & $18(54.5)$ & \\
\hline CAG repeat length, mean (SD) & $43.1(4.0)$ & \\
\hline HD diagnosis in years, mean (SD) & $6.3(7.6)$ & \\
\hline TFC, mean (SD), max $13^{\mathrm{a}}$ & $7.2(3.3)$ & \\
\hline Total score MCSI, mean (SD), $\max 26$ & & $13.5(6.6)$ \\
\hline Total score MoCA mean (SD), $\max 30^{\mathrm{b}}$ & $22.0(5.4)$ & \\
\hline Executive function, mean (SD), $\max 5$ & $3.4(1.4)$ & \\
\hline Naming, mean (SD), max 3 & $2.8(0.4)$ & \\
\hline Attention, mean (SD), $\max 6$ & $4.7(1.7)$ & \\
\hline Language, mean (SD), max 3 & $1.4(1.0)$ & \\
\hline Abstraction, mean (SD), max 2 & $1.4(0.6)$ & \\
\hline Delayed recall, mean (SD), $\max 5$ & $2.8(1.6)$ & \\
\hline Orientation, mean (SD), $\max 6$ & $5.6(0.7)$ & \\
\hline Psychotropic drugs, $N(\%)$ & $19(57.6)$ & \\
\hline Number of psychotropic drugs per patient, mean (SD) & $0.9(0.9)$ & \\
\hline Antidepressants, $N(\%)$ & $14(42.4)$ & \\
\hline SSRI, $N(\%)^{\mathrm{c}}$ & $12(36.4)$ & \\
\hline $\mathrm{TCA}, N(\%)^{\mathrm{c}}$ & $2(6.1)$ & \\
\hline Antipsychotics, $N(\%)$ & $11(33.3)$ & \\
\hline Typical antipsychotic, $N(\%)$ & $9(27.3)$ & \\
\hline Atypical antipsychotic, $N(\%)$ & $4(12.1)$ & \\
\hline Amphetamine, $N(\%)$ & $1(3.0)$ & \\
\hline Benzodiazepine, $N(\%)$ & $2(6.1)$ & \\
\hline
\end{tabular}

${ }^{\mathrm{a}}$ TFC (0-13) = The degree of independence of the patient concerning finances, domestic responsibilities, activities of the daily living, occupation and the degree of needed care. A higher score on the TFC means a more independent patient

${ }^{\mathrm{b}}$ Of the 33 MoCA's, 32 were fully available. For one MoCA, only the total score was known

${ }^{\mathrm{c}}$ Selective serotonin reuptake inhibitor (SSRI) and tricyclic antidepressant (TCA)

disturbed; on these domains the patients scored the lowest.

Next, to explore the relations between the MoCA, MCSI and other variables, a network analysis was employed. The result of our network analysis is displayed in Fig. 1. What becomes apparent from this network is that first of all, gender was not associated with any of the variables, with the exception that gender of the patient had a negative relation with gender of the caregiver. We only included patients who live with their primary caregiver, this happens to be solely man-woman dyads.

Furthermore, we observed close relationships between the other variables, as shown by the nodes representing these variables in Fig. 1. Especially CAG repeat length and time since diagnosis are central nodes within the network as reflected through their (degree, betweenness, and eigenvector) centrality scores (see Table 2). Degree centrality assesses how important a node is by counting how many 

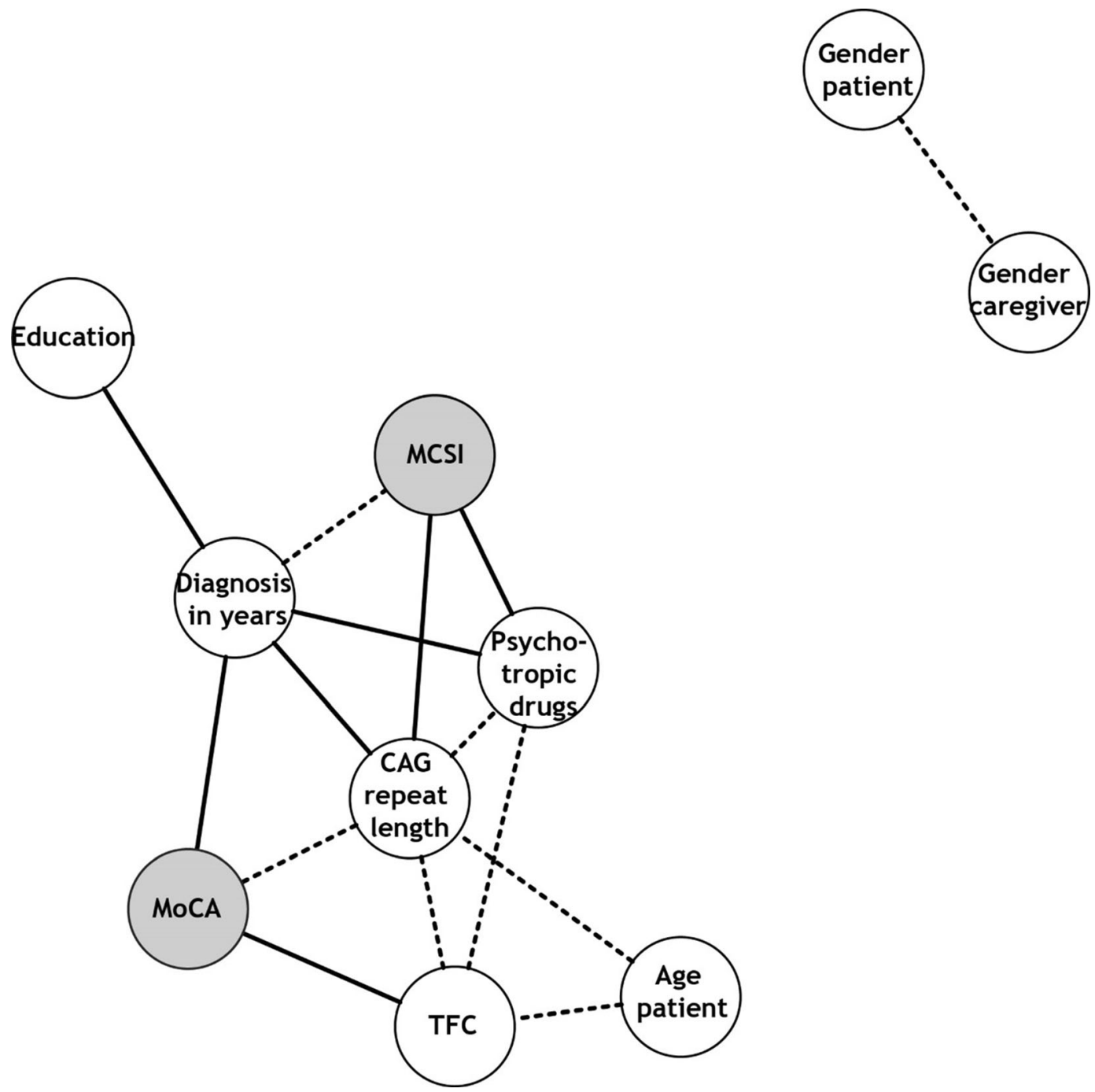

Fig. 1 Network analysis. Each node depicts a variable. A solid edge represents a positive association and a dashed edge represents a negative association

Table 2 Degree, betweenness and eigenvector centrality measures of the network. In addition, the number of positive and negative connections are listed for each node

\begin{tabular}{llllll}
\hline & Degree & Betweenness & Eigenvector & $\mathrm{nNeg}$ & $\mathrm{nPos}$ \\
\hline CAG repeat length & 6 & 6.000 & 1.00 & 4 & 2 \\
HD diagnosis & 5 & 6.833 & 0.81 & 1 & 4 \\
Psychotropic drugs & 4 & 1.167 & 0.79 & 2 & 2 \\
TFC & 4 & 1.333 & 0.71 & 3 & 1 \\
MCSI & 3 & 0.000 & 0.65 & 1 & 2 \\
MoCA & 3 & 0.667 & 0.63 & 1 & 2 \\
Age patient & 2 & 0.000 & 0.43 & 2 & 0 \\
Education & 1 & 0.000 & 0.20 & 0 & 1 \\
Gender patient & 1 & 0.000 & 0.00 & 1 & 0 \\
Gender caregiver & 1 & 0.000 & 0.00 & 1 & 0 \\
\hline
\end{tabular}


connections it has. Eigenvector centrality measures a node's importance while giving consideration to the importance of its neighbors. Betweenness centrality measures how important a node is to the shortest paths (between any pair of nodes) through the network. In all cases, the higher the centrality score, the more central a node is considered to be in the network. With respect to CAG repeat length and time since diagnosis we make the following observations from the network. Conditional on the remaining variables we observe for CAG repeat length a negative association with the MoCA, indicating that the longer the CAG repeat the lower the cognitive functioning, and a positive association with the MCSI, indicating that higher caregiver burden is associated with longer CAG repeats. Conditional on the remaining variables we observe some unexpected associations for years since diagnosis with the MoCA and MCSI, with a longer disease course associated with lower caregiver burden and higher cognitive functioning. Importantly, the largest part of the negative marginal association between MoCA and MCSI can be attributed to the mediating effects of the MCSI-years since diagnosis-MoCA and MCSI-CAG repeat length-MoCA paths. Hence, the negative association between MoCA and MCSI is mediated mostly through years since diagnosis and CAG repeat length.

Moreover, there is a conditional association between the length of the CAG repeat and the time since HD has been diagnosed, i.e. the longer the CAG repeat the longer the disease already lasted in years. There is also a positive conditional association between psychotropic drugs and MCSI, indicating that the use of psychotropic drugs is linked with a high caregiver burden. Furthermore, we observe a direct relationship between MoCA and the TFC; higher cognitive functioning is associated with more independence in daily life.

\section{Discussion}

In this study, we explored the relationship between caregiver burden and cognitive impairment of HD patients. We hypothesized a direct relation between cognitive impairment and higher caregiver burden, since most qualitative research suggested a close relationship [6-9]. However, using the network analysis, we demonstrated an indirect relationship between cognitive functioning and caregiver burden, mediated mainly by CAG repeat length and time since diagnosis. Secondly, lower cognitive functioning was related to more dependence of the patient, as reflected by TFC, and longer length of CAG repeats. We observed a relation between the use of psychotropic drugs by the patient and higher caregiver burden. Previous literature demonstrated a relationship between CAG length and cognitive functioning [11] and a relationship between cognitive functioning and caregiver burden [7,8], yet these findings have not been previously linked to each other. Here, we used the network analysis to get an insight into the mutual relationship between these variables.

\section{Caregiver Burden and Cognitive Functioning}

Our results are in line with a study by Banaszkiewicz and colleagues in which no direct relation between caregiver burden, measured by the Caregiver Burden Inventory (CBI), and cognitive functioning by means of the UHDRS cognitive scale was observed [29]. They did not investigate the indirect relations between the variables, but observed that the majority of the burden's variance was not determined by the symptoms of the patient. They postulated the important role of psychological factors in caregiver burden, such as feelings of guilt about inheritance of HD. However, Ready and colleagues did find that cognitive impairment, also assessed by the UHDRS cognitive scale, was related to a lower quality of life of caregivers [7]. Yet, in their study, quality of life was measured by one single item. It can be questioned whether this indicates the quality of life in relationship to the disease of the patient or also concerns other aspects of the caregiver's lives. Furthermore, Ready and colleagues included partners, children and other relationships, of whom a quarter did not live with the patient. Nonresident caregivers, children, parents and roommates might experience the burden differently. Further research could investigate whether the type of relationship between patient and caregiver impacts the amount of burden that the caregiver experiences from cognitive impairment. Moreover, other psychological factors that could play a role in caregiver burden can be taken into account.

The level of burden experienced by the caregivers in our study resembled the caregiver burden of HD patients in a recent study by $\mathrm{Yu}$ et al. [30]. They observed that a caregiver experienced more burden 
when they were the main caregiver (i.e. being the sole caregiver). Furthermore, a higher score on the MCSI correlated with caring for a patient with a lower TFC score. This indicated that caregiver burden was associated with more dependence of the patient. The TFC score in their study was 5.2 on average, indicating that patients were in later stages of the disease than in our population which might explain the relation found in this study. We observed that TFC was only indirectly related to caregiver burden; the relationship was mediated via psychotropic drugs, CAG repeat length and directly related to cognition. The TFC in our study had a positive correlation with MoCA. Higher cognitive functioning associated with more independence in daily life, which was in line with our expectations.

These previous studies did not assess the influence of CAG repeat length and years since HD diagnosis on the relationship between caregiver burden and cognitive functioning. Our network analysis demonstrated that these variables form important modulators.

\section{CAG Repeat Length}

We found that longer CAG repeat length was associated with a larger number of years since the HD diagnosis as well as lower cognitive functioning. Wright also demonstrated that the age of onset of HD was significantly influenced by the length of the uninterrupted CAG repeat [31]. Moreover, it is suggested the CAG repeat length plays an important role in the degree of brain atrophy in HD. The rate of atrophy in the striatal and occipito-temporo-partietal cortices are influenced by the CAG repeat length [32]. Patients with longer CAG repeat length have an increased rate of atrophy. Baake and colleagues demonstrated the association between the CAG repeat length and cognitive functioning [11]. Participants with longer $\mathrm{CAG}$ repeat length showed a more rapid cognitive decline. Furthermore, each CAG repeat length had its own curve of cognitive decline.

\section{Years Since HD Diagnosis}

We observed relations between the years since diagnosis HD on the one hand and MCSI and MoCA on the other hand. A larger number of years since HD had been diagnosed was associated with lower caregiver burden and higher cognitive functioning. This may possibly reflect an acceptance effect on the side of the patient and the caregiver or more time for both and more capacity for the patient to cope with the disease.

\section{Psychotropic Drugs}

We also found a positive relation between caregiver burden and psychotropic drug use by the patient. Neuropsychiatric symptoms like depression, irritability and psychosis are often present in HD and regularly lead to pharmacological therapy [33]. The cognition of the HD patients was not directly affected by using psychotropic drugs in our sample, which is in line with a review by Dickey and La Spada showing that none of the investigated psychotropic drugs improved cognition [15].

\section{Limitations}

This is a retrospective and cross-sectional study and has its accompanying limitations. Because the data are not collected longitudinally, the causality of the associations is unclear. Retrospective studies are more prone to missing data that cannot be completed and the researchers have no influence on the quality of the data. Because of the cross-sectional perspective of this study it is unclear whether the neuropsychiatric symptoms or the psychotropic drug use were leading to higher caregiver burden. Aspects of psychotropic drug use such as side effects or the medication-related checkups could lead to a higher caregiver burden. Caregiver characteristics or coping style might also play a role in the above-mentioned relation, but these were not investigated in this study.

Moreover, we have a relatively small sample size $(\mathrm{N}=33)$ due to the inclusion and exclusion criteria. Yet, we ensured that we had patient-partner dyads and that our assessments were performed on the same day.

Furthermore, we only included caregiving partners who live with the patient. Since we believed caregiving burden might especially be high for partners in the home situation. Non-resident caregivers, children, parents and roommates might experience the burden differently.

Finally, the MCSI is a reliable and useful instrument to measure the strain of caregivers [22]. However, it is unclear whether the height of the score corresponds with the same level of burden for each caregiver. It is possible a substantial inter-individual 
variation occurs in the answers. The items can be appreciated differently in all kinds of situations and moods. Furthermore, the separate items do not necessarily have the same value to individual caregivers. Regarding the MoCA, according to a review by Mestre, this tool was more sensitive to cognitive dysfunction in HD than the Mini-Mental State Examination. Yet, they also state that there is no golden standard regarding the screening of cognition in HD and that the assessment is considered brief with only a limited number of items per domain [34]. Further research could assess cognitive performance using more elaborate cognitive test batteries.

Implications for Rehabilitation Practitioners

Our results show that caregiver burden was affected by the time since the patient was diagnosed with HD. Rehabilitation practitioners should keep in mind that both the patient and the caregiver may experience changes since the diagnosis. As our results show the impact on the caregiver burden, we recommend to discuss caregiver burden with the caregivers on a yearly basis, considering the changing nature of $\mathrm{HD}$ and to support the caregivers according to their needs.

Living at home for as long as possible is important for patient, caregiver and society. A low caregiver burden is amongst other things essential for the patient to live at home. As our study demonstrated, a longer CAG repeat length and short time since HD diagnosis are indicators for caregiver burden. These should be assessed and the patient and the caregiver treated or supported where and if possible.

We found no direct effect of psychotropic drug use on the cognition of the HD patients. In the rehabilitation setting, psychotropic drugs should be a topic discussed with patients and their caregivers and prescribed according to the guidelines.

\section{Conclusion}

In this study, we demonstrated the possible importance of CAG repeat length and time since HD diagnosis as mediators between cognition and caregiver burden. A longer CAG repeat length and short time since HD diagnosis are identifiers for caregiver burden. These factors should be indicators for assessing the caregivers and their burden more extensively. An association between caregiver burden and psychotropic drug use was found which could focus subsequent studies on the direction of this association and characteristics of the patients and their caregivers. Psychotropic drug use is an important topic which should be discussed with caregivers when addressing the burden of caring for HD patients.

The network analysis provides insights on the direct and indirect associations between our variables of interest. This method produces comprehensible results and we encourage its use in future research. This study could be a stepping-stone to more comprehensive research on the components that make up caregiver burden in HD.

Acknowledgements We would like to thank the staff of the outpatient HD clinic (Noorderbreedte Care group) for their input and cooperation.

Funding This study received no funding.

\section{Compliance with Ethical Standards}

Conflict of interest The authors declare that they have no conflict of interest.

Ethics Approval Ethics approval was not applicable, since the measures were part of the standard treatment and care protocol. Furthermore, patients and caregivers are informed in writing about the usage of their data for research purposes. They can refuse this at any time, the refusal will be noted in the electronic dossier and patients will be excluded from participation in research. The data used for research was pseudonymized.

Open Access This article is licensed under a Creative Commons Attribution 4.0 International License, which permits use, sharing, adaptation, distribution and reproduction in any medium or format, as long as you give appropriate credit to the original author(s) and the source, provide a link to the Creative Commons licence, and indicate if changes were made. The images or other third party material in this article are included in the article's Creative Commons licence, unless indicated otherwise in a credit line to the material. If material is not included in the article's Creative Commons licence and your intended use is not permitted by statutory regulation or exceeds the permitted use, you will need to obtain permission directly from the copyright holder. To view a copy of this licence, visit http://creativecommons.org/licenses/by/4.0/. 


\section{References}

1. Baig SS, Strong M, Quarrell OWJ. The global prevalence of Huntington's disease: a systematic review and discussion. Neurodegener Dis Manag. 2016;6(4):331-43.

2. The Huntington's Disease Collaborative Research Group. A novel gene containing a trinucleotide repeat that is expanded and unstable on Huntington's disease chromosomes. Cell. 1993;72(6):971-83.

3. Tarolli CG, Chesire AM, Biglan KM. Palliative care in Huntington disease: personal reflections and a review of the literature. Tremor Other Hyperkinetic Movements (New York, N.Y.). 2017;7:454.

4. Domaradzki J. The impact of Huntington disease on family carers: a literature overview. Psychiatr Pol. 2015;49(5):931-44.

5. Røthing M, Malterud K, Frich JC. Caregiver roles in families affected by Huntington's disease: a qualitative interview study. Scand J Caring Sci. 2014;28(4):700-5.

6. Aubeeluck AV, Buchanan H, Stupple EJN. 'All the burden on all the carers': exploring quality of life with family caregivers of Huntington's disease patients. Qual Life Res. 2012;21(8):1425-35.

7. Ready RE, Mathews M, Leserman A, Paulsen JS. Patient and caregiver quality of life in Huntington's disease. Mov Disord. 2008;23(5):721-6.

8. Simpson JA, Lovecky D, Kogan J, Vetter LA, Yohrling GJ. Survey of the Huntington's disease patient and caregiver community reveals most impactful symptoms and treatment needs. J Huntington's Dis. 2016;5(4):395-403.

9. Williams JK, Skirton H, Paulsen JS, Tripp-Reimer T, Jarmon L, McGonigal Kenney M, et al. The emotional experiences of family carers in Huntington disease. J Adv Nurs. 2009;65(4):789-98.

10. Røthing M, Malterud K, Frich JC. Balancing needs as a family caregiver in Huntington's disease: a qualitative interview study. Health Soc Care Community. 2014;23(5):569-76.

11. Baake V, Reijntjes RHAM, Dumas EM, Thompson JC, REGISTRY Investigators of the European Huntington's Disease Network, Roos RAC. Cognitive decline in Huntington's disease expansion gene carriers. Cortex. 2017;95:51-62.

12. Paulsen JS, Miller AC, Hayes T, Shaw E. Cognitive and behavioral changes in Huntington disease before diagnosis. In: Feigin AS, Anderson KE, editors. Handbook of clinical neurology, vol. 144. Amsterdam: Elsevier; 2017. p. 69-91.

13. Papoutsi M, Labuschagne I, Tabrizi SJ, Stout JC. The cognitive burden in Huntington's disease: pathology, phenotype, and mechanisms of compensation. Mov Disord. 2014;29(5):673-83.

14. Vinther-Jensen T, Larsen IU, Hjermind LE, Budtz-Jørgensen E, Nielsen TT, Nørremølle A, et al. A clinical classification acknowledging neuropsychiatric and cognitive impairment in Huntington's disease. Orphanet J Rare Dis. 2014;9:114.

15. Dickey AS, La Spada AR. Therapy development in Huntington disease: from current strategies to emerging opportunities. Am J Med Genet Part A. 2018;176(4):842-61.
16. Goldberg JF, Ernst CL. Managing the side effects of psychotropic medications. 2nd ed. Washington: American Psychiatric Association; 2019.

17. Sackley C, Hoppitt TJ, Calvert M, Gill P, Eaton B, Yao G, Pall H. Huntington's disease: current epidemiology and pharmacological management in UK primary care. Neuroepidemiology. 2011;37(3-4):216-21.

18. Zarowitz BJ, O'Shea T, Nance M. Clinical, demographic, and pharmacologic features of nursing home residents with Huntington's disease. J Am Med Dir Assoc. 2014;15(6):423-8.

19. Borsboom D, Cramer AO. Network analysis: an integrative approach to the structure of psychopathology. Annu Rev Clin Psychol. 2013;9:91-121.

20. Veenhuizen RB, Kootstra B, Vink W, Posthumus J, van Bekkum P, Zijlstra M, Dokter J. Coordinated multidisciplinary care for ambulatory Huntington's disease patients. Evaluation of 18 months of implementation. Orphanet $\mathbf{J}$ Rare Dis. 2011;6:77.

21. Simpson SA, Rae D. A standard of care for Huntington's disease: who, what \& why. Neurodegener Dis Manag. 2012;2(1):1-5.

22. Thornton M, Travis SS. Analysis of the reliability of the modified caregiver strain index. j Gerontol Ser B. 2003;58(2):127-32.

23. Nasreddine ZS, Phillips NA, Bédirian V, Charbonneau S, Whitehead V, Collin I, et al. The Montreal Cognitive Assessment, MoCA: a brief screening tool for mild cognitive impairment. J Am Geriatr Soc. 2005;53(4):695-9.

24. Huntington Study Group. Unified Huntington's Disease Rating Scale: reliability and consistency. Mov Disord. 1996;11(2):136-42.

25. Epskamp S, Cramer AOJ, Waldorp LJ, Schmittmann VD, Borsboom D. qgraph: Network visualizations of relationships in psychometric data. J Stat Softw. 2012;48(4):1-18.

26. Van Wieringen WN, Peeters CFW. Ridge estimation of inverse covariance matrices from high-dimensional data. Comput Stat Data Anal. 2016;103:284-303.

27. Liu H, Lafferty J, Wasserman L. The Nonparanormal: semiparametric estimation of high-dimensional undirected graphs. J Mach Learn Res. 2009;10:2295-328.

28. Kamada T, Kawai S. An algorithm for drawing general undirected graphs. Inf Process Lett. 1989;31:7-15.

29. Banaszkiewicz K, Sitek EJ, Rudzińska M, Soltan W, Slawek J, Szczuldlik A. Huntington's disease from the patient, caregiver and physician's perspectives: three sides of the same coin? J Neural Transm. 2012;119:1361-5.

30. Yu M, Tan K, Koloms K, Bega D. Assessment of caregiver burden in Huntington's disease. J Huntington's Dis. 2019;8:111-4.

31. Wright GEB, Collins JA, Kay C, McDonald C, Dolzhenko E, Xia Q, et al. Length of uninterrupted CAG, independent of polyglutamine size, results in increased somatic instability, hastening onset of Huntington disease. Am J Hum Genet. 2019; 104:1116-26.

32. Johnson EB, Ziegler G, Penny W, Rees G, Tabrizi SJ, Scahill RI, et al. Dynamics of cortical degeneration over a decade in Huntington's Disease. https://www.biorxiv.org/ content/biorxiv/early/2019/02/07/537977.full.pdf (2019). Accessed 8 Sept 2019. 
33. Van Duijn E. Medical treatment of behavioral manifestations of Huntington disease. In: Feigin AS, Anderson KE, editors. Handbook of clinical neurology, vol. 144. Amsterdam: Elsevier; 2017. p. 129-39.

34. Mestre TA, Bachoud-Lévi AC, Marinus J, Stout JC, Paulsen JS, Como P, et al. Rating scales for cognition in
Huntington's disease: critique and recommendations. Mov Disord. 2018;33(2):187-95.

Publisher's Note Springer Nature remains neutral with regard to jurisdictional claims in published maps and institutional affiliations. 